\title{
Voltage Stability Assessment Using Neural Networks in The Deregulated Market Environment
}

\author{
*Diponkar Paul ${ }^{1},{ }^{2}$ Md. Sadiq iqba, ${ }^{3}$ Mohammed Ibrahim Hussain and ${ }^{4}$ A. H. M. Shahriar parvez.
}

\begin{abstract}
The project was done in Nanyang technological university, Singapore at Electric power research Lab during the period from Sep 2006 to Nov 2007. The liberalization of electric market has generated the unbundling of large utilities into separated generation, Transmission and Distribution companies with subsequent changes in the operating condition at electric power systems. Most of the breakdowns in the electric power systems are caused by unfavorable dynamic response of the networks following system disturbances. In addition, environmental and economic considerations are forcing power systems to be operated closer to their limits of stability .Therefore dynamic security assessment of power systems is becoming increasingly important. Voltage collapse is one of the instability which occurs when the system is heavily loaded. In addition, the mechanism of voltage collapse is due to the load reacting to the voltage changed which leads to further voltage changes. In this project, indicators utilizing the generator-load mismatch and active/reactive power margins for on-line assessment of proximity of voltage instability of power system is to be developed. The approach utilizes an artificial neural networks(ANN) function approximator. In the area of Power systems, problems may be expressed in different ways depending upon their nature. The problem formulation may be expressed in terms of complex systems, say nonlinear, large scale, dynamical, discrete, stochastic, ransom like quasi-periodical, time-variant parameter systems etc. Among these factors, the nonlinear and the large scale systems make power system problems more hard to solve. Apart from linear systems, no good analytical system are available for the complicated problems. ANN is a good promising candidate for dealing with them.
\end{abstract}

Index Terms-SA, GA, SE, , PV curve, TO.

\section{INTRODUCTION}

The primary purpose of the system generation control is to balance the total system generation against load demands and losses, so that the desired frequency and power interchange with neighboring systems (tie flows) is maintained. In this structure, in addition to the controllers operating directly on individual system elements such as the generators, there is usually some form of overall plant controller that coordinates the controls of closely linked elements. The plant controllers are in turn supervised by system controllers at the operating centers. The system controller actions are coordinated by

\footnotetext{
${ }^{12}$ Assistant Professor \& senior member of IACSIT, 34Senior Lecturer, ${ }^{1}$ Department of Electrical \& Electronic Engineering,

${ }^{234}$ Department of computer science and engineering, Bangladesh University

*Corresponding author's Email: DIPO0001@ntu.edu.sg,
}

pool level master controllers. (O. I. Elgerd,, 1971)The control system is thus highly distributed and relies on many different types of telemetering and control signals. Supervisory controls and data acquisition system provides information to indicate the system status. Firstly the analysis of the load data with respect to the quality of the data and with respect to the different consumer behavior depending on seasons, week days and holidays, secondly the estimation of the load to be forecasted based on previously experienced load demands. The self organizing feature may be successfully solved the analysis task by creating classes of load patterns which are averages of several similar load patterns. Choosing the 24 hourly loads, next day peak load and 4 different day typed as inputs Basic requirements for the normal operation of a power system is that the total load must equal the total generation at any time. That reserve generation must also be scheduled in order to account for the load uncertainty and possible outages of generation plant. The reserve capacity that is spinning, synchronized and ready to take up load is generally known as spinning reserve. Some utilities include only this spinning reserve in their assessment of system adequacy, whereas others also include one or more or the following factors, interruptible loads, assistant from interconnected systems, voltage/frequency reduction. These additional factors add to the effective spinning reserve and the total entity is known as operating reserve. Historically operating reserve requirements for a power system have been done. The most frequently and method being a spinning generating reserve equal to one or largest unites in operating. Probabilistic spinning reserve assessment is to determine the required spinning reserve capacity for the specific reliability indices. For a power system with $\mathrm{N}$ components, the total number of the system states with $2^{\mathrm{N}}$. For example, a system with nine components will have a total of 512 states. A power system usually consists of thousands of components. There will be a great number of system states for a larger power system. The computing time required may be very long. It is also noticed from practical experiences that the probability of three and more components failing at same time is very small. It is required to practice to limit the number of states by selecting the contingencies which have larger probability of occurrence and neglecting. Those contingencies which have a probability of occurrences less than a predefined minimum valuec the iteration is to cut the list of the events and reduce the calculation. System security corresponds to the ability of the power system to withstand some unforeseen but probable, disturbances, with the minimal description of service or its 
quality. In the area of system planning, the issue of reliability plays an important role. System security is the counterpart of system reliability in the field of power system operation. In reliability analysis one is given the configuration of the system and the probability distribution of individual component failures. Reliability assessment translates this information into reliability measures (K.R.W.Bell, A.R.Daniels and R.W.Dunn, 1993). The planning process consists of adding components (i.e. generating units) or reconfiguring the system in order to meet accepted reliability standards. Since the analysis is done in a planning mode, these options can be implemented in due to the field current limits. The major causes are uncontrolled machine flux

decay dynamics that force possible cascading voltage collapse by reduction of voltage behind transient reactance. However the voltage instability mechanisms due to an interaction between the machine field flux dynamics and load have not yet been fully reported so far. The voltage instability mechanism related high load demand is investigated when synchronous generator is under normal excitation. First, the voltage unstable phenomenon at maximum laudability limits with synchronous machine under manual and automatic excitations are explored. Secondly, the mechanism which gives rise to voltage instability due to a significant change of the flux linkage characteristic is discussed. Finally, the emphasis is given to explore performance limitation in the excitation control, caused by open loop unstable pole. The management and procurement of the reactive power from the generation resource that are entitled to provide VAR as an ancillary service in the current electricity markets are very crucial in maintaining system security and reliability. Presently there are several payment schemes being used for the remuneration of the VAR service providers. Although there is no standard methodology by which the transmission operation in these markets pay. VAR resources, the cost based pricing is commonly used in most of those payment structures, where the long-term contract basis is the preferred choice of transmission operators to mitigate the potential exercise of market power that would be associated with inadequate number of regional VAR resources,. These pricing methods emphasize mostly the recovery of embedded costs, lost of opportunity costs and some other additional small amount operational costs "costs of transmission losses and operational of voltage regulators" incurred by the generation facilities for providing VAR service. While the portion of the generator embedded costs attributed to reactive power is very complicated to separate from total generator capital costs, the operational costs are very difficult to quantify and the operational costs are very difficult to quantify and the determination of opportunity costs is not straightforward is often affected by specific market arrangements. This implies that the generator VAR costs are not accurately allocated and consequently the current cost based pricing mechanisms rely to much extent on heuristics and operator's judgment to procure VAR service. Another thing is that the VAR providers should be remunerated based on their value, which reflects the relative importance of VAR sources in maintaining system security and reliability. The main factors affecting the value of an individual VAR source are its location, system configurations, operating conditions etc. They have no direct reliability

with the previous embedded and variable costs. One possible way that would be used to quantify the worth of a particular VAR supplier is to assess its relative competitiveness in liberalized market of reactive power. Several researches have realized this fact and have attempted to address the procurement of VAR service in a competitive market and achieve the economical efficiency of this vital service(T. V. Cutsem and C. Yournas,, 1998). The most important physical limiting factor in the ability of a generator to provide reactive power support is its generation capability limit. It represents the hard physical limitation of a generator's capability for the simultaneous production of real and reactive power. It is clear that the reactive power limits that the generator can produce depend on the active power output, where the limits of the field current, armature current and the under excitation of the generator are the boundaries that restrict the joint production of real and reactive power. This expression is known as lot opportunity cost. The generators are obligated to provide a certain amount of reactive power without any payment of compensation from the TO. This VAR amount expressed as $\mathrm{Q}_{\mathrm{md} 1}$ in the lagging power factor region and $\mathrm{Q}_{\mathrm{md} 2}$ in the leading power factor region as shown in Figure 1 , where $\mathrm{Q}_{\mathrm{md} 1}$ and $\mathrm{Q}_{\mathrm{md} 2}$ are determined based on the principle of the proportional obligations that compel the generators to provide VAR service in proportion to their active power output. Accordingly, the VAR of a generator beyond the mandatory is considered solely as an ancillary service that the generator offers and should be compensated for providing it. The provided ancillary service VAR is divided into two parts. The first part is the VAR injection or absorption that the generator provides beyond its mandatory without rescheduling its real power output and the second part is the lost of opportunity cost incurred as a result of reducing its real power output. Region / $\left(\mathrm{Q}_{\mathrm{md} 2}\right.$ to $\mathrm{Q}_{\mathrm{md} 1)}$ : The reactive power produced in this region is obligatory with no payment. Region // $\left(\mathrm{Q}_{\mathrm{md} 1}\right.$ to $\mathrm{Q}_{1}$ and $\mathrm{Q}_{\mathrm{md} 2}$ to $\mathrm{Q}_{2}$ ): This region represents the extra reactive $\mathrm{VAR}$ provided by generator beyond its obligatory without rescheduling its real power output. A generator in this region is expecting a payment from the TO for its service. Region // $\left(\mathrm{Q}_{\mathrm{md} 1}\right.$ to $\mathrm{Q}_{1}{ }^{*}$ and $\mathrm{Q}_{\mathrm{md} 2}$ to $\mathrm{Q}_{2}{ }^{*}$ ): In this region the generator will reduce its real power output and consequently its lost revenue will be recovered by $\mathrm{TO}$ (Transmission operators) (D.E Runelhart and J.C McClelland (Eds),1998).

\section{Methodology:}

A P-V curve method which is a traditional static voltage stability analysis method is a kind of computation analysis method based on the physical conception. Setting the result of the basic system power flow, increasing the system load gradually, and calculating the system voltage corresponding to each operating point, a series of $(\mathrm{P}, \mathrm{V})$ points reflecting the relationship between the load power actually absorbed and the node voltage can be obtained and a P-V curve can be formed. The inflection point of $\mathrm{P}-\mathrm{V}$ is considered as the voltage stability curve, the area above the inflection point is the voltage stability region and the area below is considered 
as the voltage instability region. The distance from the current system operating point to the inflection point(Y. Kataoka,1994) is known as the system voltage stability region. For a definite five bus power system we can use the Newton Raphson method in multi bus system. The transfer limit surface of a power system was investigated. We specifically and explicitly defined the transfer limit surface when there are constraints. The smoothness of the transfer limit surface, taking into account the generator reactive power limits. With strict proof were not presented, the following properties were derived with at least a certain degree of logical rigor. These properties were verified through a small scale numerical example-The transfer limit surface of a single constraint power system is smooth. The transfer limit surface of a power system with two or more constraints is in general non smooth. The degree of smoothness is not expected to be serious. Hence one can expect that applications based on a normal vector are valid even for systems with constraints. The methodology adopted and the above result can form a basis for engineering applications related to the transfer limit surface.

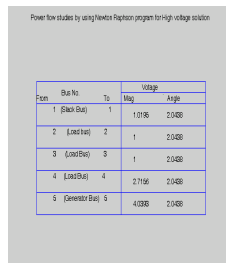

(a)

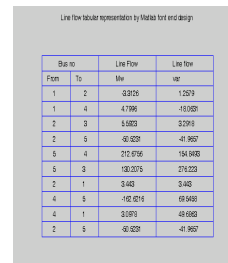

(b)

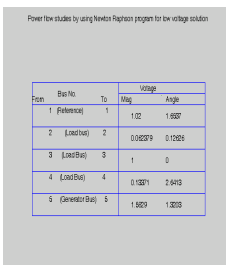

(c)
Figure 1: (a)Generalized power Flow studies by using Newton Raphson program for high voltage solution in per unit (b)Generalized power flow studies by using Newton Raphson program for line flow in per unit.(c )Generalized power flow studies by using Newton Raphson program for low voltage solution.

The voltage stability has already become a serious concern to power system operators. It is willing to find out a fast assessment method to estimate the static voltage stability level. To the dispatcher, the most practical index for analyzing the voltage security is power margin. So it is necessary to determine the critical point of the steady state voltage collapse accurately. There are important parameters to access the capability of each node to maintain the voltage stability, i.e. the critical voltage and the power limit at the loading point. The static voltage stability analysis has been widely used in the analysis for the real life power system. But theoretically, static analysis is not restrict because there is certainly some error to take the maximum power transfer point as the critical point of the voltage stability, it is necessary to find out how much the error is and

whether it is still meaningful to use the static analysis method in the analysis of the voltage stability. The error of the load power value and the critical voltage taking the inflection point of the P-V curve as the static voltage stability limit in the static voltage can be calculated. Standard IEEE9 system is analyzed and explained as an example. Finally draw the following facts:-It is reasonably and feasible to take the P-V inflection point as the system voltage instability. Taking the $\mathrm{P}-\mathrm{V}$ inflection point as the critical point of system voltage instability in static voltage analysis, the error is different for different load model. It is the biggest under the load model of the "permanent power". The less advantage of the content power in the load model, the smaller the error is. Avoid selecting "the permanent power" load model as far as possible in order to reduce the analysis error in the static voltage stability analysis. In the actual analysis application, it is definitely acceptable for the errors of system critical load power within $2 \%$ and the critical voltage within $0.05 \%$. So it reasonable and feasible to take the $\mathrm{P}-\mathrm{V}$ inflection point as the system voltage instability point. While the system reaches to the limit of the steady state stability, Jacobian matrix becomes singular, and there is a zero eigenvalue. During the transient period from the normal operating node to the stability limit, Jacobian will tend to be singular and the corresponding minimum eigenvalue is then considered as the measure to the steady-state voltage margin.

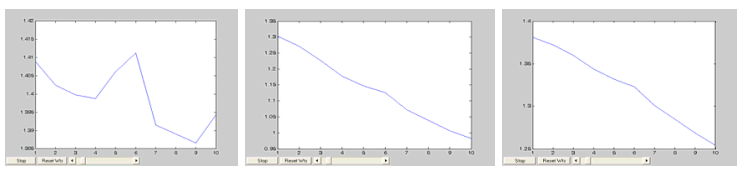

Figure 2: Evaluation of $\partial \mathrm{P}_{\mathrm{k}} / \partial \mathrm{V}_{\mathrm{k}}$ the load buses 2, 3 and 4 respectively.

The $\mathrm{y}$ axis represents $\partial \mathrm{P}_{\mathrm{k}} / \partial \mathrm{V}_{\mathrm{k}}$ and $\mathrm{x}$ axis represents $\lambda$ as a function of the loading factor where $\mathrm{k}$ is the slack bus. The loading range was from the base case to the vicinity of the MLP. The smooth close-to-linear behavior noted here is valid for all tested systems. Before neural network can gain the necessary recognization as useful problem solving tools in the power industry, certain fundamentals issues needed to be addressed. Some of them are associated with neural network technology, and others are problem dependent such as :Selection of neural network architecture and learning algorithm( Feser, K., Braun, U., Engler, F., Maier, A, 1991), such as net size, learning step, number of training patterns and iterations based on the problem dynamics. Determining the proper consistency of the training and testing data sets including the range of training data, the spanning of the data in the operational space, and the statistical properties of the data. Formulation of features that provide high correlation between the training pattern and the desired $\mathrm{NN}$ response. The feature may have to be relatively insensitive to variations in topology, operation, and control characteristics of the system through the use of averaging, scaling and time gradient techniques(T. Hiyama,, T. Sameshima and C.M. Lim, 1991). Statistical feature selection techniques to the dimensionality of the input data while preserving classification accuracy. This would complement the higher level feature selection that may have already been performed through expert knowledge.Ability of the NN to generalize within the operational span of the system. The NN should first be able to learn the input pattern rather than memorizing them. The ability of the NN is to maintain its weights in a viable region without causing a large number of neurons to saturate. The ability of a NN to converge to an acceptable minimum within a specified range of weights.In neural key applications, the NN must be adaptable to slow changes in the system dynamics, and be able to update itself adaptively.NN inversion with Queries to enhance class boundaries and improve the data distribution in the operating space. 


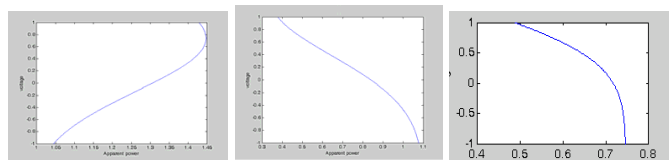

Figure 3: :PV curve for stability margin for load buses 2,3 and 4 respectively.

In small disturbance analysis, dynamic characteristics of the generators, reactors and speed governors are taking into consideration. The system operation operating point corresponding to the eigenvalu when the eigen-root locus of the system static matrix passes through the vertical or imaginary axis is the right point corresponding to the voltage collapse. Here compares the maximum load power and the critical voltage corresponding to the P-V curve's inflection point with maximum load power and the critical point of voltage collapse state matrix passes through the vertical or imaginary axis. To contrast the two power flow points, the concept of load power deviation coefficient and the voltage deviation are introduced. As a result, the error of the load power value and the critical voltage taking the inflection point of the P-V curve as the static voltage stability limit in the static voltage stability analysis. P-V curve method which is a traditional static traditional static voltage stability analysis method is a kind of computation analysis method based on the physical conception. Setting the result of the basic power flow, increasing the system load gradually, and calculating the system voltage corresponding to each operating point, a series of $(\mathrm{P}-\mathrm{V})$ points reflecting the relationship between the load power actually absorbed and a $\mathrm{P}-\mathrm{V}$ curve can be formed. The reflection point of the P-V curve is considered as the voltage stability point, the area above the inflection point is the voltage stability region. The distance from the current system operating point to the inflection point is known as the system voltage stability margin.

reduce
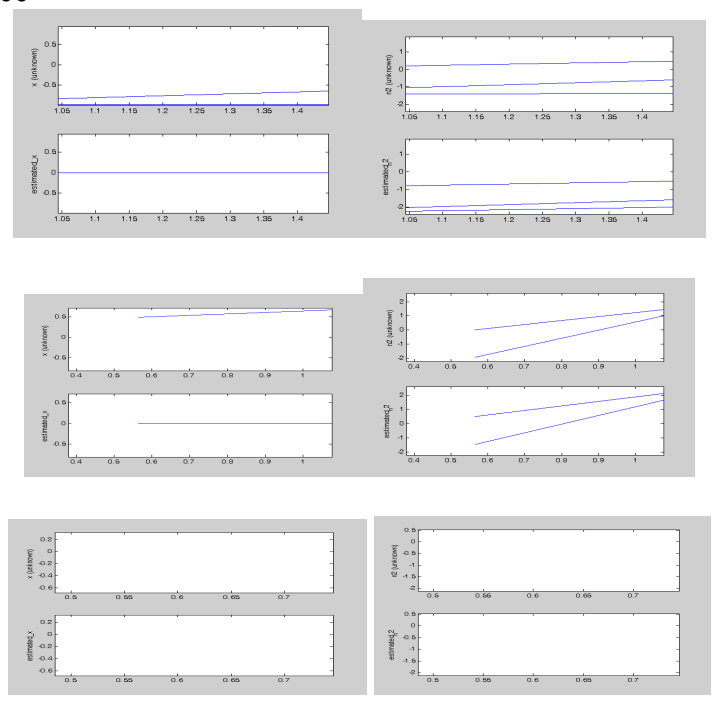

Figure 4: Noise elimination curve for both estimated_n2 and n2(unknown).for load buses 2,3 and 4 respectively.

When information on decisions is incomplete or the reliability is uncertain, the fuzzy logic can be a very helpful tool. The fuzzy logic theory has been applied to several areas including neural networks. Fuzzy logic is mainly used with $\mathrm{NN}$ to increase the dynamic range of the output neurons and reduce the effect of data noise. The supervised neural networks are trained with extracted feature and fuzzified targets. After the network is trained, the response of the network is defuzzified. The above entire system is described in the following block diagram shown in Figure 5.

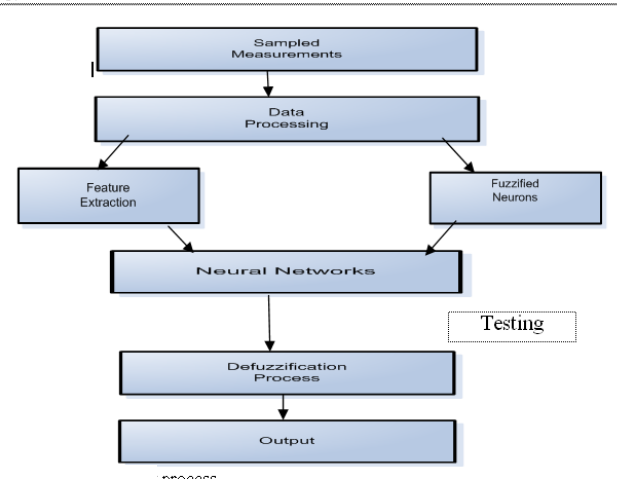

Figure 5:The fuzzified neural network process.

In the actual analysis application, it is desired definitely acceptable for the errors of system critical load power within $2 \%$ and the critical voltage within $0.05 \%$. So it is reasonable and feasible to take the p-v inflection point as the system voltage instability point. The static voltage stability analysis method has been widely used in the analysis for the real life power system. But theoretically, static analysis method is not restrict because there is certainly some error to take the maximum power transfer point as the critical because there is certainly some error to take the maximum power transfer as the critical point of the voltage stability. It is necessary to find out how much the error is and whether it is still meaningful to use the static analysis method in thep-v curve compares the maximum load power and the critical voltage corresponding to the p-v curve's inflection point with the maximum load power and the critical point of voltage collapse corresponding to the eigen value point when the eigen locus of the static matrix passes through the vertical or imaginary axis. To compare the two power flow points, the concept of load power deviation coefficient and the voltage deviation are introduced. As a result of the error of the load power value and voltage and the critical voltage taking the inflection point of the $p$-v curve as the static voltage stability limit in the static voltage stability analysis can be calculated.

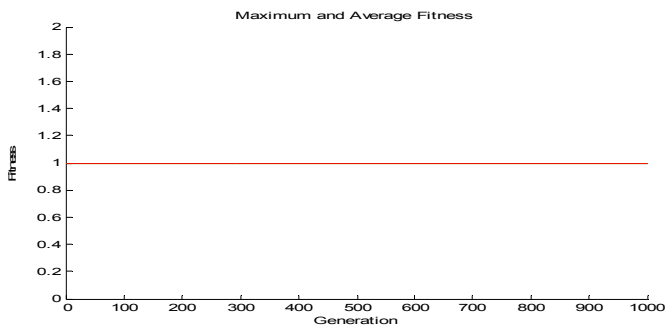

Fig 6: Average fitness of the population of solution.

Since our goal is to enhance the performance of Neural Networks, the emphasis is put on the ancillary role of genetic algorithm in the Neural Network computation. The control actions found by the generic algorithm are checked with a full 
Newton Raphson load flow calculation. The fitness values shown here were normalized and a value of 1 presents the theoretical case that no constraints are violated. The fitness of the best solution in the population increased considerably faster. Fig 7 presents the penalty of the constraints and the number of the controls used in the fitness of the best solution. Since the goal of the search process is first of all to find a set of control action that eliminates all constraints violations, the weight factor $m_{1}$ should be chosen sufficiently large in comparison with $\mathrm{m}_{2 s}$. In order to ensure that a single $0.1 \mathrm{p} . \mathrm{u}$ voltage constraint violation will cause a heavier penalty that $\mathrm{K}$ control action do, $\mathrm{m}_{1}<10000^{*} \mathrm{k}^{*} \mathrm{~m}_{2}$ should be chosen. In the first generation created by the GA(Genetic Algorithm), (A.G.Bakirtzis, P. N. Biskas, C. E. Zoumas and V. Petridis, 2002)the constraint violations will be minimized and eventually become zero, when and if a solution for the control problem is found. At this stage, the algorithm may be stopped., since the primary goal of the search process is reached. However it is possible to continue with the search process and the population of solutions will develop according to the last term of the fitness, which is the term expressing the number of controls used(K. Iba, , 1994). Unacceptable large changes of control variables are avoided by constraints in the GA. The initial population of solutions may be chosenanalysis of voltage stability(Y. Kataok, 1992). The above

at random, as is traditionally done. However a careful choice of the initial population can improve the efficiency in a substantial way. In view of the fact that a solution consisting of a small number of control actions is preferred, a part of the initial population is selected to preserent "one control action" solution. For a case of participating controls, the first $\mathrm{i}=1 \ldots \mathrm{k}$ strings represent a single step increase in control i, while all other controls remain unchanged. Step size is the smallest possible, according to the coding of the variable. A similar procedure is followed for the next $\mathrm{k}$ strings, representing a decrease in each of the control values. In total $2 \mathrm{k}$ strings are selected, and the others are randomly chosen.

\section{CONCLUSION:}

Most of the ANN applications to SA and SE, reported in the literature, are of explanatory nature. They attempt to determine the feasibility of using different ANN architectures and determine which inputs and types of training produces the most accurate results. This study can be considered to be similar to the selection of the good selection of security indices or approximate system performance (ASP) models for SA. That is finding what ASP is fast and accurate. Although the computational speed is a non issue when using ANNs training time is a crucial issue in power system security assessment. The experience acquired from the development of the ANN based model strongly indicates that the ANN technology has matured enough to be applied successfully in many power system problems. However the success will eventually depend on the ability to remove a major obstacle(Thomas Kyte, 2006, 2006) ; at present there is neither a firm theory or nor even a set heuristic guidelines or procedures to assist the developer to design neural networks.
The very flexibility that makes the ANN technology so amenable to data modeling also creates burden for the developers to select a 'good' if not the 'best' combination of topology, learning rules and parameters, activation functions, thresholds and input variables. At the present time, there is no complete theoretical basis to relate the above the parameters to known characteristics of the system that is being modeled. The lack of complete set of systematic design procedure constitutes the main obstacle to the practical use of neural networks. Any further work will produce lasting results in the area if it also recognizes the fact the ANN technology can hardly provide all the modeling capabilities needed in a real life application. Consequently, any progress in ANN design should allow for integration of this technology with other modeling techniques from the statistical arema and especially with other technologies from the artificial intelligent are.

\section{ACKNOWLEDGEMENT}

I would like to acknowledge Dr. Lie Tek Tjing, Associate professor, school of Electrical and Electronic engineering, Nanyang technological University, Singapore during that period for supervising the power engineering degree. I would also thank Assoc professor Dr. Chen Shium in that school for power quality course on that contemporary. I am grateful to Dr. Md. Hamidul Haque, Associate Professor in that school during that period for his suggestion and voltage stability course.

\section{REFERENCES}

[1] Y. Kataoka, "A Rapid Solution for the Maximum Loading Point of a Power System”, Elect. Eng. Japan, Vol. 114, no. 2, pp 34-47, 1994.

[2] Y. Kataoka, "An Approach for the Regulation of a Power Flow Solution Round the Maximum Loading Point", IEEE Transactions on Power Systems,. Vol. 7, No 3, pp. 1068-1077, Aug. 1992.

[3] K. Iba, "Reactive Power Optimization by Genetic Algorithm," IEEE Trans. Power Systems, Vol, 9, No.2, pp. 685-692, May, 1994.

[4] T. Hiyama,, T. Sameshima and C.M. Lim, "Fuzzy Logic Stabilizer with Digital Compensator for Stability

[5] Enhancement of Multi Machine Power Systems", Proc. of third symposium on. Expert System Application

[6] to Power Systems (ESAPS), Tokyo and Kobe, Japan, pp 455-461, 1991.

[7] Feser, K., Braun, U., Engler, F., Maier, A., “ Application of Neural Network in Busbar Numerical Protection Systemss(NBPS), "Proceedings of the first international Forum on Applications of Neural Networks to power system, Seattle, WA July 23-26, 1991, 117-121.

[8] D.E Runelhart and J.C McClelland (Eds), Parallel Distributed Processing Explorations in the Microstructure of Cognition. Vol 1. MIT Press, Cambridge, MA, 1986, pp. 318-362, USA.

[9] Thomas Kyte, 2006. Programming and of Oracle 9I \& 10g. Designing: people's post and telecommunication publishing.

[10] O. I. Elgerd, "Electric Energy systems Theory: An Introduction", McGraw Hill, 1971. USA.

[11] T. V. Cutsem and C. Yournas, "Voltage Stability System of Electric Power Systems", Kluwer, Norwell, MA, USA, 1998.

[12] K.R.W.Bell, A.R.Daniels and R.W.Dunn, "Modeling of operator heuristics in dispatch for security enhancement." IEE Trans power syst., vol. 13, no3, pp. 937-945,Aug 1993.

[13] A.G.Bakirtzis, P. N. Biskas, C. E. Zoumas and V. Petridis, "Optimal power flow by enhanced genetic algorithm," IEEE Trans Power Syst., vol 17, no.2, pp. 229-136, May 2002.

[14] C Liu and K. Tomsovic, "An expert system assisting decision making reactive power/voltage control,” IEEE Trans Power Syst. Vol. 1, no. 3, pp. 195-201, Aug 1986. 


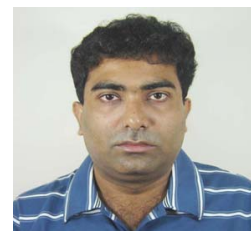

First and correspondence author: Diponkar Paul is currently serving as Assistant Professor in department of Electrical and Electronic engineering at Bangladesh university after passing his master degree. He is having qualifications: B.Sc. Engg., DISM, M.Sc. Engg. His field of interest is in the area of energy conversions, power system modeling and advanced control theories covering the application of IT to instrumentations. From Oct 2004 to July 2006, he was working as Lecturer in the department of Computer science and engineering at Pundra university of science and technology, Bogra. In Singapore during his master degree studies at Nanyang technological university he was involved in financial service operations integrated to IT administration job from Dec 2006 to Feb 2008. 